Mathematical Modelling And Analysis

Volume 19 Number 2, April 2014, 145-154

http://dx.doi.org/10.3846/13926292.2014.908792

(c) Vilnius Gediminas Technical University, 2014
Publisher: Taylor\&Francis and VGTU

http://www.tandfonline.com/TMMA

Print ISSN: 1392-6292

Online ISSN: 1648-3510

\title{
Nonlocal Third Order Boundary Value Problems with Solutions that Change Sign*
}

\section{Sergey Smirnov}

\author{
Institute of Mathematics and Computer Science, University of Latvia \\ Raina bulvaris 29, LV-1459 Riga, Latvia \\ E-mail: srgsm@inbox.1v
}

Received September 10, 2013; revised March 1, 2014; published online April 15, 2014

\begin{abstract}
We investigate the existence and the number of solutions for a third order boundary value problem with nonlocal boundary conditions in connection with the oscillatory behavior of solutions. The combination of the shooting method and scaling method is used in the proofs of our main results. Examples are included to illustrate the results.
\end{abstract}

Keywords: nonlinear boundary value problems, nonlocal boundary conditions, estimation of the number of solutions.

AMS Subject Classification: 34B15.

\section{Introduction}

In this paper we consider the nonlocal boundary value problem consisting of the nonlinear third order differential equation

$$
x^{\prime \prime \prime}=-p(t) f(x)
$$

and the boundary conditions

$$
x(0)=x^{\prime}(0)=0, \quad x(\eta)=x(1),
$$

where $0<\eta<1, f: \mathbb{R} \rightarrow \mathbb{R}$ and $p: \mathbb{R} \rightarrow[0, \infty), p(t) \not \equiv 0$ are continuous. We assume throughout that

(H1) $x f(x)>0$ for $x \neq 0$;

(H2) there exist $m, M>0$ such that $|f(x)|>M$ when $|x|>m$;

(H3) $\int_{t_{0}}^{+\infty} p(s) d s=\infty, \int_{-\infty}^{t_{0}} p(s) d s=\infty$.

These assumptions provide the oscillatory behavior of solutions of equation $(1.1)$.

\footnotetext{
* This research has been supported by the Latvian Council of Science project 34512012.
} 
We also use the following assumptions

(H4) $p(B t)=B^{k} p(t)$ for some $k \geq 0$ and all $B>0$;

(H5) $f(B x)=B^{q} f(x)$ for some $q>1$ and all $B>0$.

An example of equation (1.1), which satisfies conditions (H1), (H2), (H3), (H4) and (H5) is $x^{\prime \prime \prime}=-t^{2} x^{3}$.

The special case $f(x)=|x|^{q} \operatorname{sgn} x, q>1$ is of a particular interest. In fact, the Emden-Fowler type differential equation

$$
x^{\prime \prime \prime}+p(t)|x|^{q} \operatorname{sgn} x=0
$$

is a prototype of (1.1). Therefore, equation (1.1) is a generalization of the second order Emden-Fowler equation

$$
u^{\prime \prime}+t^{\nu}|u|^{\mu} \operatorname{sgn} u=0
$$

where $\nu, \mu$ are real constants. Such equations appear in the problems of polytropic gas spheres of finite radius or finite mass $[4,8]$. The study of EmdenFowler equation (1.4) has been one of the main objects in the field of nonlinear analysis in recent years since the appearance of the monograph due to R. Bell$\operatorname{man}[2]$.

Boundary value problems with nonlocal conditions are an important and actual field of research since they appear in physics and other areas of applied mathematics, and as a consequence, have generated a lot of interest over the years. Survey of known results can be found in the monograph by S.K. Ntouyas [6]. Results concerning $n$th order nonlocal boundary value problems were obtained by P.W. Eloe and B. Ahmad [3], J.R. Graef and T. Moussaoui [5]. In [3], applying the fixed point theorem in cones, the authors establish the existence of at least one positive solution of the problem

$$
\begin{gathered}
u^{(n)}+a(t) f(u)=0, \quad t \in(0,1), \\
u(0)=u^{\prime}(0)=\cdots=u^{(n-2)}=0, \quad \alpha u(\eta)=u(1),
\end{gathered}
$$

where $0<\eta<1,0<\alpha \eta^{n-1}<1$, if $f$ is either superlinear or sublinear. In [5] the authors discuss the existence of both sign changing solutions and positive solutions for problem (1) under conditions different from those imposed in [3]. Some recent contributions to the study of second order nonlinear boundary value problems with nonlocal conditions include, for example, the papers of S. Pečiulyté and A. Štikonas [7], N. Sergejeva [9]. In [7] the authors consider the Sturm-Liouville problem with various types of nonlocal two-point boundary conditions. In [9] the author establishes the solvability results for the boundary value problem with nonlocal conditions based on the knowledge of the spectrum for the auxiliary problem.

In this paper, we are interested in the existence and the number of sign changing solutions for problem (1.1), (1.2). Our approach in the estimation of the number of solutions is based on the combination of the shooting method and scaling method. 
Analogous technique was used by the author [10] to provide results on the estimation of the number of solutions to the boundary value problem for equation (1.1) subject to the two point conditions $x(0)=x^{\prime}(0)=0, x(1)=0$.

In view of the use of the shooting method, the problem the author faced with is the non-continuability of the solutions. For example, the function

$$
x(t)=(105 / 8)^{\frac{1}{2}}\left(t-t_{0}\right)^{-\frac{3}{2}}
$$

is a non-continuable solution with vertical asymptote $t=t_{0}$ of the equation $x^{\prime \prime \prime}=-x^{3}$ defined for $t>t_{0}$. Also, such kind equations can have oscillating type non-continuable solutions. Results concerning non-continuability of solutions of the third order nonlinear differential equations can be found in [1]. However, we will show that the non-continuability does not influence the results on estimation of the number of solutions to boundary value problem.

The paper is organized as follows. Section 2 contains some auxiliary results. Section 3 is devoted to the oscillatory properties of solutions of equation (1.1). In Section 4 we consider dependence of zeros of solutions on initial data. In Section 5 we deal with the number of solutions to boundary value problem (1.1), (1.2). Also one example is given to illustrate the results. In order the paper to be self-contained, we provide some auxiliary results, published in [10] with proofs.

\section{Preliminary Results}

First, let us discuss the relationship between assumptions (H1), (H2), (H3), (H4) and (H5).

Proposition 1. If a function $f(x)$ satisfies assumptions ( $\mathrm{H} 1)$ and (H5), then $f(x)$ satisfies assumption (H2) also.

Proof. Let us choose $0<x_{1}<x_{2}$. Obviously, there exists a $B>1$ such that $x_{2}=B x_{1}$. Now consider $f\left(x_{2}\right)=f\left(B x_{1}\right)=B^{q} f\left(x_{1}\right)$. Since $0<x_{1}$ and $x f(x)>0$, then $B^{q} f\left(x_{1}\right)>f\left(x_{1}\right)$ and $f\left(x_{2}\right)>f\left(x_{1}\right)$. Thus, $f(x)$ is strictly increasing function for $x>0$. Analogously we can show that $f(x)$ is strictly increasing for $x<0$. It is obviously, that increasing function satisfies assumption (H2).

Remark 1. We state condition (H2) because some propositions below hold if only (H1) and (H2) are satisfied for some function $f(x)$.

Proposition 2. If a function $p(t)$ satisfies assumption $(\mathrm{H} 4)$, then $p(t)$ satisfies assumption (H3) also.

Proof. First, assume $k>0$. We can obtain that the function $p(t)$ is increasing for $t>0$ and is decreasing for $t<0$ repeating the arguments used in the proof of the previous proposition.

Next, assume $k=0$. Since for every $B>0 p(B t)=B^{0} p(t)=p(t)$, it follows that $p(t)=$ Const $>0$.

Evidently, that in both cases condition (H3) holds. 
Remark 2. We state condition (H3) because some propositions below hold if only (H3) is satisfied for some function $p(t)$.

Next, consider auxiliary results, which we will use in the proofs of the oscillatory behavior for solutions of equation (1.1).

Proposition 3. Suppose $x(t) \in C^{3}(I)$. If $x(a) \geq 0, x^{\prime}(a) \leq 0, x^{\prime \prime}(a) \geq 0$ (but not all zero) and $x^{\prime \prime \prime}(t) x(t)<0$ when $x(t) \neq 0$, then $x(t)>0, x^{\prime}(t)<0$, $x^{\prime \prime}(t)>0$ for $t<a$.

Proof. Let $x(a) \geq 0, x^{\prime}(a) \leq 0, x^{\prime \prime}(a) \geq 0$ and $(x(a))^{2}+\left(x^{\prime}(a)\right)^{2}+\left(x^{\prime \prime}(a)\right)^{2}>0$.

In all cases $x(t)$ will be positive in some open interval with the right boundary point $t=a$. Suppose that there exists a point $t=t_{0}$ such that $x\left(t_{0}\right)=0$ and $x(t)>0$ for $t_{0}<t<a$. Since $x^{\prime \prime \prime}(t) x(t) \leq 0$, it follows that $x^{\prime \prime \prime}(t)<0$ for $t_{0}<t<a$. Consider

$$
x^{\prime \prime}(t)=x^{\prime \prime}(a)-\int_{t}^{a} x^{\prime \prime \prime}(s) d s, \quad t_{0} \leq t \leq a .
$$

The right-hand side is positive, and increases as $t \searrow t_{0}$, as long as $x^{\prime \prime \prime}(t)$ remains negative. We thus conclude that $x^{\prime \prime}(t)$ is positive for $t_{0} \leq t<a$.

Consider

$$
x^{\prime}(t)=x^{\prime}(a)-\int_{t}^{a} x^{\prime \prime}(s) d s, \quad t_{0} \leq t \leq a .
$$

The right-hand side is negative, and decreases as $t \searrow t_{0}$, as long as $x^{\prime \prime}(t)$ remains positive. We thus conclude that $x^{\prime}(t)$ is negative for $t_{0} \leq t<a$.

Consider

$$
x(t)=x(a)-\int_{t}^{a} x^{\prime}(s) d s, \quad t_{0} \leq t \leq a
$$

The right-hand side is positive, and increases as $t \searrow t_{0}$, as long as $x^{\prime}(t)$ remains negative. We thus conclude that $x(t)$ is positive for $t_{0} \leq t<a$. These contradictions prove the proposition.

Corollary 1. Suppose $x(t) \in C^{3}(I)$. If $x(a) \leq 0, x^{\prime}(a) \geq 0, x^{\prime \prime}(a) \leq 0$ (but not all zero) and $x^{\prime \prime \prime}(t) x(t)<0$ when $x(t) \neq 0$, then $x(t)<0, x^{\prime}(t)>0, x^{\prime \prime}(t)<0$ for $t<a$.

Proof. The proof follows from Proposition 3 considering $y(t)=-x(t)$.

Remark 3. The function $x(t)$ from Proposition 3 and Corollary 1 may be assumed to be a solution of differential equation (1.1).

\section{Oscillatory Properties of Solutions}

An application of Proposition 3 and Corollary 1 leads to the following results. 
Corollary 2. Assume that condition (H1) is satisfied. If $x(t)$ is a nontrivial solution of (1.1), $x(a)=x(b)=0$ and $a<b$, then $x^{\prime}(b) \neq 0$ (a simple zero cannot exist on the left of a double zero).

Proof. Let $x^{\prime}(b)=0$, and, without loss of generality, let $x^{\prime \prime}(b)>0$. In view of (H1) $x^{\prime \prime \prime}(t) x(t)<0$. Then, by Proposition $3 x(t)>0$ for $t<b$. But $x(a)=0$, $a<b$. The contradiction proves the corollary.

Corollary 3. Suppose that condition (H1) holds. If $x(t)$ is a nontrivial solution of $(1.1)$ and $x(a)=x(b)=0(a<b)$, then $x^{\prime}(b) x^{\prime \prime}(b)>0$.

Proof. Assume $x^{\prime}(b) x^{\prime \prime}(b) \leq 0$. In view of $(\mathrm{H} 1) x^{\prime \prime \prime}(t) x(t)<0$. If $x^{\prime}(b)<0$, $x^{\prime \prime}(b) \geq 0$, then, by Proposition $3 x(t)>0$ for $t<b$. We have a contradiction, since $x(a)=0$. If $x^{\prime}(b)>0, x^{\prime \prime}(b) \leq 0$, then, by Corollary $1 x(t)<0$ for $t<b$. We have a contradiction, since $x(a)=0$. In view of Corollary $2, x^{\prime}(b) \neq 0$.

Proposition 4. Let $x(t)$ be a solution of equation (1.1) such that $x(a)=$ $x^{\prime}(b)=0(a<b), x(t) \neq 0$ for $t \in(a, b)$. If condition (H1) is fulfilled, then $x(t)$ vanishes in $(b,+\infty)$.

Proof. Assume that $x(t)$ does not change sign for $t>b$. Without loss of generality, let $x(t)>0, t>b$. Then $x(t)>0$ for $t \in(a, b)$. Multiplying equation (1.1) by $x(t)$ and integrating from $a$ to $t$, we obtain

$$
\int_{a}^{t} x(s) x^{\prime \prime \prime}(s) d s=-\int_{a}^{t} x(s) p(s) f(x(s)) d s .
$$

Integrating the first term by parts, we get

$$
x(t) x^{\prime \prime}(t)-x(a) x^{\prime \prime}(a)-\int_{a}^{t} x^{\prime \prime}(s) x^{\prime}(s) d s=-\int_{a}^{t} x(s) p(s) f(x(s)) d s,
$$

or

$$
x(t) x^{\prime \prime}(t)=\frac{1}{2} x^{\prime 2}(t)-\frac{1}{2} x^{\prime 2}(a)-\int_{a}^{t} x(s) p(s) f(x(s)) d s .
$$

If $t=b$ we obtain

$$
x(b) x^{\prime \prime}(b)=\frac{1}{2} x^{\prime 2}(b)-\frac{1}{2} x^{\prime 2}(a)-\int_{a}^{b} x(s) p(s) f(x(s)) d s<0 .
$$

First of all exclude the case $x(b)=0$. If $x(b)=0$, then a simple zero exists on the left of a double zero, which contradicts Corollary 2. So $x(b)>0$, then $x^{\prime \prime}(b)<0$. Since $x(t)>0$, then (in view of (H1) and (1.1)) $x^{\prime \prime \prime}(t)<0$ and $x^{\prime \prime}(t)$ is strictly decreasing. Thus, $x^{\prime \prime}(t)<0$ for $t>b$ and $x^{\prime}(t)$ is strictly decreasing for $t>b$. Since $x^{\prime}(b)=0$ and $x^{\prime}(t)$ is strictly decreasing for $t>b$, then $x^{\prime}(t)<0$ for $t>b$. Thus, $x(t)$ is strictly decreasing for $t>b$. If two consecutive derivatives of $x(t)$ are negative then $x(t)$ must ultimately be negative. This completes the proof of the proposition. 
Proposition 5. Let $x(t)$ be a solution of equation (1.1) such that $x(a)=0$. If conditions $(\mathrm{H} 1),(\mathrm{H} 2)$ and $(\mathrm{H} 3)$ hold, then $x(t)$ vanishes in $(a,+\infty)$.

Proof. Suppose that $x(t)$ does not vanish for $t>a$. Without loss of generality, let $x(t)>0$ for $t>a$. If there exists $b>a$ such that $x^{\prime}(b)=0$, then the proof follows from Proposition 4 above. Therefore, assume that $x^{\prime}(t)$ does not vanish for $t>a$. Since $x^{\prime}(t)>0$ for $t$ immediately to the right of $a$, it follows that $x^{\prime}(t)>0$ for $t>a$. As $x(t)>0$, then (in view of (H1) and (1.1)), $x^{\prime \prime \prime}(t)<0$ and $x^{\prime \prime}(t)$ is strictly decreasing.

First suppose there exists $t_{1} \geq a$ such that $x^{\prime \prime}\left(t_{1}\right)=0$. Then $x^{\prime \prime}(t)<0$ for $t>t_{1}$. If two consecutive derivatives of $x^{\prime}(t)$ are negative then $x^{\prime}(t)$ must ultimately be negative.

Now assume that $x^{\prime \prime}(t)>0$ for $t>a$. So $x^{\prime}(t)$ is strictly increasing for $t>a$. Integrating equation (1.1) between $t_{0}>a$ and $t$ we obtain

$$
\int_{t_{0}}^{t} x^{\prime \prime \prime}(s) d s=-\int_{t_{0}}^{t} p(s) f(x(s)) d s
$$

or eliminating nonnegative terms and using assumption (H2) we get

$$
x^{\prime \prime}\left(t_{0}\right)=x^{\prime \prime}(t)+\int_{t_{0}}^{t} p(s) f(x(s)) d s \geq \int_{t_{0}}^{t} p(s) f(x(s)) d s \geq M \int_{t_{0}}^{t} p(s) d s .
$$

The left side is independent of $t$ and thus the integral on the right hand side must converge as $t \rightarrow+\infty$. This contradiction proves the proposition.

Remark 4. The condition $x(a)=0$ in Proposition 5 is essential, because there exist solutions (for example (1.6)) of equation (1.1) which do not vanish.

Corollary 4. Assume that conditions (H1), (H2) and (H3) fulfil. If $x(t)$ is a nontrivial solution of (1.1) and $t=a$ is a zero of $x(t)$, then $x(t)$ has an infinity of simple zeros in $(a,+\infty)$. If $t=a$ is a double zero of $x(t)$, then $x(t)$ does not vanish in $(-\infty, a)$.

Proof. Proposition 5 implies that after any zero of $x(t)$ must follow another zero. At the same time, according to Corollary 2 there is no zero to the left of a double zero. Hence the proof.

\section{Scaling Formula}

Proposition 6. Suppose that conditions (H4) and (H5) are fulfilled. If $x(t)$ is a solution of equation (1.1), then the function

$$
y(t)=B^{\frac{3+k}{q-1}} x(B t),
$$

where $B>0$ is an arbitrary constant, is also a solution of equation (1.1).

Remark 5. A similar statement for higher order Emden-Fowler type autonomous equation can be found in [1]. 
Proof. The proposition can be proved by direct substitution. So

$$
y^{\prime \prime \prime}(t)=B^{\frac{3+k}{q-1}+3} x^{\prime \prime \prime}(B t), \quad f(y(t))=B^{\frac{3+k}{q-1} q} f(x(B t)) .
$$

Then

$$
\begin{aligned}
B^{\frac{3+k}{q-1}+3} x^{\prime \prime \prime}(B t) & =-p(t) B^{\frac{3+k}{q-1} q} f(x(B t)) \\
& =-p(B t) B^{-k} B^{\frac{3+k}{q-1} q} f(x(B t))=B^{-k} B^{\frac{3+k}{q-1} q} x^{\prime \prime \prime}(B t) .
\end{aligned}
$$

Therefore

$$
B^{\frac{3+k}{q-1}+3}=B^{-k} B^{\frac{3+k}{q-1} q}, \quad \text { or } \quad B^{\frac{3 q+k}{q-1}}=B^{\frac{3 q+k}{q-1}} .
$$

Hence the proof.

Proposition 7. Suppose that conditions (H4) and (H5) are fulfilled. If $x\left(t, \beta_{0}\right)$ is a solution of equation (1.1), such that

$$
x\left(0, \beta_{0}\right)=x^{\prime}\left(0, \beta_{0}\right)=0, \quad x^{\prime \prime}\left(0, \beta_{0}\right)=\beta_{0} \neq 0,
$$

then every solution $x(t, \beta)$ of equation (1.1) which has a double zero at $t=0$ and the second derivative $\beta$ at $t=0$ of the same sign as $\beta_{0}\left(\beta \beta_{0}>0\right)$ can be expressed via solution $x\left(t, \beta_{0}\right)$ as

$$
x(t, \beta)=\left(\beta / \beta_{0}\right)^{\frac{3+k}{1+k+2 q}} x\left(\left(\beta / \beta_{0}\right)^{\frac{q-1}{1+k+2 q}} t, \beta_{0}\right) .
$$

Remark 6 . We distinguish the cases of $x^{\prime \prime}$ having opposite signs at $t=0$ in Proposition 7, because if $x(t)$ is a solution of $(1.1)$, then $(-x(t))$ need not to be a solution of (1.1). As an example we present the equation

$$
x^{\prime \prime \prime}=- \begin{cases}x^{3}, & x \geq 0, \\ 2 x^{3}, & x<0,\end{cases}
$$

where $f(x)$ satisfies condition (H5) with $q=3$.

Proof. The proof follows from Proposition 6 and direct substitution. So

$$
x^{\prime \prime}(0, \beta)=B^{\frac{3+k}{q-1}+2} \beta_{0}=\beta .
$$

Thus $B=\left(\beta / \beta_{0}\right)^{(q-1) /(1+k+2 q)}$. The proof is complete.

\section{Number of Solutions for Boundary Value Problem}

Proposition 8. Assume that conditions (H1), (H2) and (H3) are satisfied. Let $x\left(t, \beta_{0}\right)$ be a nontrivial solution of equation (1.1) with the initial conditions $x(0)=0, x^{\prime}(0)=0, x^{\prime \prime}(0)=\beta_{0}$. For every $0<\eta<1$ the equation

$$
x\left(t, \beta_{0}\right)=x\left(\eta t, \beta_{0}\right)
$$

has a countable set of zeros $\tau_{i}>0, i=1,2, \ldots$. 
Remark 7 . The values $x\left(\tau_{i}, \beta_{0}\right)=x\left(\eta \tau_{i}, \beta_{0}\right)$ depend on the value of $0<\eta<1$.

Proof. The proof follows from the fact that $x\left(t, \beta_{0}\right)$ has an infinity of simple zeros in $(0,+\infty)$ (Corollary 4$)$.

Now we are ready to prove our main result.

Theorem 1. Suppose that conditions (H1), (H4) and (H5) are fulfilled, then boundary value problem (1.1), (1.2) has a countable set of solutions $y_{i}(t)$ with $y_{i}^{\prime \prime}(0)>0$ and a countable set of solutions $u_{i}(t)$ with $u_{i}^{\prime \prime}(0)<0, i=1,2, \ldots$

Proof. Let $x\left(t, \beta_{0}\right)$ be a nontrivial solution of equation (1.1) with the initial conditions $x(0)=0, x^{\prime}(0)=0, x^{\prime \prime}(0)=\beta_{0}$.

By Proposition 7

$$
y(t, \beta)=\left(\beta / \beta_{0}\right)^{\frac{3+k}{1+k+2 q}} x\left(\left(\beta / \beta_{0}\right)^{\frac{q-1}{1+k+2 q}} t, \beta_{0}\right)
$$

is a solution of equation (1.1) with initial data $y(0)=y^{\prime}(0)=0, y^{\prime \prime}(0)=\beta$. Next, consider the function

$$
y(\eta t, \beta)=\left(\beta / \beta_{0}\right)^{\frac{3+k}{1+k+2 q}} x\left(\left(\beta / \beta_{0}\right)^{\frac{q-1}{1+k+2 q}} \eta t, \beta_{0}\right) .
$$

Let $t=1$, we get

$$
y(1, \beta)=\left(\beta / \beta_{0}\right)^{\frac{3+k}{1+k+2 q}} x\left(\left(\beta / \beta_{0}\right)^{\frac{q-1}{1+k+2 q}}, \beta_{0}\right)
$$

and

$$
y(\eta, \beta)=\left(\beta / \beta_{0}\right)^{\frac{3+k}{1+k+2 q}} x\left(\left(\beta / \beta_{0}\right)^{\frac{q-1}{1+k+2 q}} \eta, \beta_{0}\right) .
$$

By Proposition 8 for every $0<\eta<1$ there exist $\tau_{i}(i=1,2, \ldots)$ such, that

$$
x\left(\tau_{i}, \beta_{0}\right)=x\left(\eta \tau_{i}, \beta_{0}\right), \quad i=1,2, \ldots
$$

Obviously, we can choose $\beta=\beta_{i}(i=1,2, \ldots)$ such, that $\left(\beta_{i} / \beta_{0}\right)^{\frac{q-1}{1+k+2 q}}=\tau_{i}$. Thus, there exists a countable set of values $\beta_{i}$ such, that

$$
x\left(\left(\beta_{i} / \beta_{0}\right)^{\frac{q-1}{1+k+2 q}}, \beta_{0}\right)=x\left(\left(\beta_{i} / \beta_{0}\right)^{\frac{q-1}{1+k+2 q}} \eta, \beta_{0}\right) .
$$

Therefore, the equation $y(1, \beta)=y(\eta, \beta)$ has countable set of zeros $\beta_{i}(i=$ $1,2, \ldots)$. Hence the proof.

Remark 8. Theorem 1 remains valid if boundary conditions (1.2) are replaced by more general ones

$$
x(0)=0, \quad x^{(i)}(0)=0, \quad x^{(j)}(\eta)=x^{(j)}(1), \quad i \in\{1,2\}, j \in\{0,1,2\} .
$$

Example 1. Consider the problem

$$
x^{\prime \prime \prime}=-t^{2} x^{3}, \quad x(0)=x^{\prime}(0)=0, \quad x(1 / 2)=x(1) .
$$




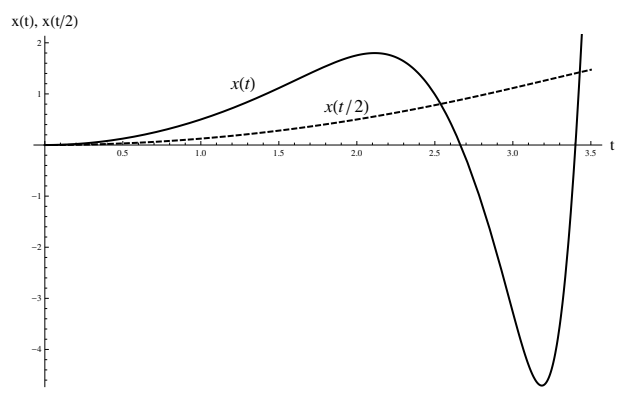

Figure 1. Functions $x(t)$ (solid) and $x(t / 2)$ (dashed).

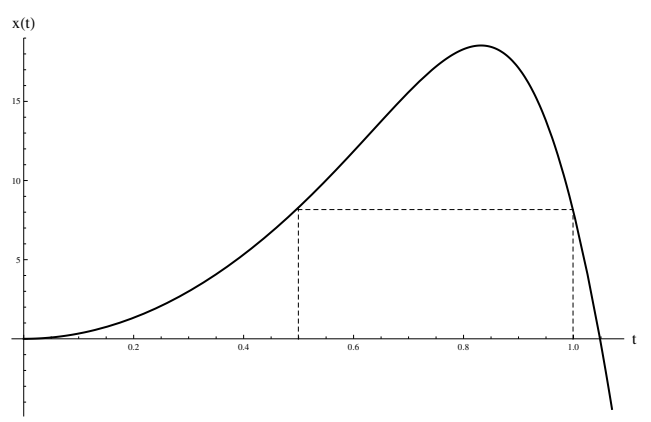

Figure 2. Solution $x_{1}(t)$ of problem (5.2).

Conditions (H1), (H4) and (H5) are fulfilled, then boundary value problem (5.2) has a countable set of solutions.

Let us construct first two solutions $x_{1}(t)$ and $x_{2}(t)$ of problem (5.2) by using the technique, described in the proof of Theorem 1 . So, consider the auxiliary initial value problem

$$
\begin{aligned}
& x^{\prime \prime \prime}=-t^{2} x^{3}, \\
& x(0)=x^{\prime}(0)=0, \quad x^{\prime \prime}(0)=1
\end{aligned}
$$

and denote its solution by $x(t)$. Then, consider the function $x(t / 2)$ and find first two zeros $\tau_{1}$ and $\tau_{2}$ of the equation $x(t)=x(t / 2)$ (see Figure 1 ). Thus, by using numerical simulation, we obtain $\tau_{1} \approx 2.542, \tau_{2} \approx 3.431$. Next, find the values of the second derivative $\beta_{1}$ and $\beta_{2}$ for solutions to boundary value problem (5.2) from the equation $\beta^{9 / 2}=\tau_{i}, i=1,2$. So, $\beta_{1} \approx 66.578, \beta_{2} \approx 256.681$. Solutions $x_{1}(t)$ and $x_{2}(t)$ of boundary value problem (5.2) are presented in Figure 2 and Figure 3 .

\section{Acknowledgments}

The author wishes to express his thanks to the referee for very valuable suggestions and careful corrections. 


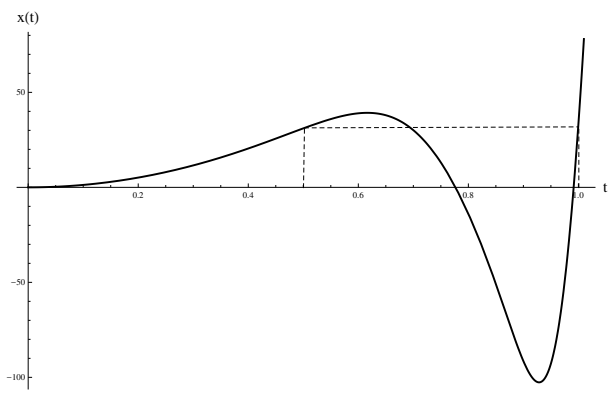

Figure 3. Solution $x_{2}(t)$ of problem (5.2).

\section{References}

[1] I.V. Astashova. Application of dynamical systems to the study of asymptotic properties of solutions to nonlinear higher-order differential equations. J. Math. Sci., 126:1361-1391, 2005. http://dx.doi.org/10.1007/s10958-005-0066-6.

[2] R. Bellman. Stability Theory of Differential Equations. McGraw-Hill, New York, 1953.

[3] P.W. Eloe and B. Ahmad. Positive solutions of a nonlinear $n$th order boundary value problem with nonlocal conditions. Appl. Math. Lett., 18:521-527, 2005. http://dx.doi.org/10.1016/j.aml.2004.05.009.

[4] R. Emden. Gaskugeln. Leipzig, 1907.

[5] J.R. Graef and T. Moussaoui. A class of $n$ th-order BVPs with nonlocal conditions. Comput. Math. Appl., 58:1662-1671, 2009. http://dx.doi.org/10.1016/j.camwa.2009.07.009.

[6] S.K. Ntouyas. Nonlocal initial and boundary value problems: a survey. In A. Cañada, P. Drábek and A. Fonda(Eds.), Handbook of Differential Equations, volume 2 of Ordinary Differential Equations, pp. 461-558, North Holland, 2005. Elsevier.

[7] S. Pečiulytè and A. Štikonas. Sturm-Liouville problem for stationary differential operator with nonlocal two-point boundary conditions. Nonlinear Anal. Model. Control, 11(1):47-78, 2006.

[8] G. Sansone. Equazioni differenziali nel campo reale. Parte seconda. Bologna, 1949 .

[9] N. Sergejeva. The regions of solvability for some three point problem. Math. Model. Anal., 18(2):191-203, 2013. http://dx.doi.org/10.3846/13926292.2013.780189.

[10] S. Smirnov. On some spectral properties of third order nonlinear boundary value problems. Math. Model. Anal., 17(1):78-89, 2012. http://dx.doi.org/10.3846/13926292.2012.645076. 